\title{
artigo
}

Fernandes, D.M.A.P.; Melo, V.F.C.; Assis, L.M.B.; Pinheiro, M.L.; Morais, M.M.M.; Batista, M.C.A.R.

A insustentável leveza do toque: reconhecimento do cuidado ao pé diabético

\section{A insustentável leveza do toque: reconhecimento do cuildado ao pé diabético}

The delicacy of a touch: taking care of the diabetic foot

La insostenible ligereza del tacto: reconocimiento del cuidado del pie diabético

\begin{abstract}
RESUMO
Diabetes mellitus é um problema de saúde pública, onde o cuidado com o pé é essencial, a fim de reduzir as complicações macro e microvasculares. Objetivo: Visa abordar a importância da avaliação dos pés diabéticos na prevenção de complicações e da educação em saúde na potencialização do autocuidado, a partir da vivência de residentes em Medicina de Família e Comunidade. Método: Trata-se de um estudo observacional, descritivo e transversal. Resultado: Mesmo com recursos escassos, pode-se restaurar peles e almas de pessoas que não possuíam autocuidado ou que não procuravam assistência à saúde. A longitudinalidade como uma das características da Atenção Primária à Saúde permitiu uma melhora do acesso aos usuários e de sua qualidade de vida. Conclusão: A educação em saúde dos portadores de pé diabético contribui significativamente para diminuição de agravos. As expectativas dos profissionais e dos pacientes com doenças crônicas tornam o cuidado desafiador.
\end{abstract}

DESCRITORES: Atenção Primária à Saúde; Pé Diabético; Educação em Saúde.

\section{ABSTRACT}

Diabetes mellitus is a public health issue, in which taking care of the feet is essential, in order to reduce the micro and macro vascular complications. Objective: This study aims to talk about the importance of examining diabetic feet to prevent bad outcomes and of health education increasing the self-care routine, through the Family Practice medical residents' experience. Method: It is an observational, descriptive and transversal report. Result: Even without enough resources, it was possible to restore not only the skins but these patients' souls. The longitudinality as one of the characteristics of the Primary Health Care provided an improvement in the access and also in the quality of life quality. Conclusion: The health education in these cases contributes to avoid damage in chronic diseases, and the patient and the professionals' expectations make the follow-up challenging.

DESCRIPTORS: Primary Health Care; Diabetic Foot; Health Education.

\section{RESUMEN}

La diabetes mellitus es un problema de salud pública, donde el cuidado de los pies es fundamental para reducir las complicaciones vasculares. Objetivo: Tiene como objetivo abordar la importancia del pie diabético en la prevención de complicaciones y la educación para la salud en la potenciación del autocuidado, a partir de la experiencia de los residentes en Medicina Familiar y Comunitaria. Método: Se trata de un estudio observacional, descriptivo y transversal. Resultado: Incluso con recursos escasos, la piel y el alma de estas personas se puede recuperar a través de la longitudinalidad como una de las características de la Atención Primaria de Salud, permitiendo un mejor acceso a los usuarios y su calidad de vida. Conclusión: La educación sanitaria para personas con pie diabético contribuye a reducir los problemas de salud. Las expectativas de los profesionales y los pacientes con enfermedades crónicas hacen que la atención sea un desafío.

DESCRIPTORES: Atención Primaria de Salud; Pie Diabético; Educación en Salud.

RECEBIDO EM: 27/11/2020 APROVADO EM: 03/12/2020

\section{Denise Mota Araripe Pereira Fernandes}

Mestranda em Saúde da Família pela FACENE, médica de família e comunidade, docente e preceptora da Faculdade de Ciências Médicas da Paraíba/PB).

ORCID: 0000-0001-7804-882X

\section{Vilma Felipe Costa de Melo}

Doutora. Psicóloga. Orientadora e docente do Mestrado em Saúde da Família pela FACENE.

ORCID: 0000-0001-5721-3240 


\section{Lana Muriely Borges de Assis}

Residente em Medicina de Família e Comunidade (MFC) pela Secretaria Municipal de Saúde de João Pessoa - SMS/JP em parceria com a Faculdade de Ciências Médicas da Paraíba (FCM-PB). Graduada em medicina pela Faculdade de Medicina Nova Esperança (FAMENE).

ORCID: 0000-0002-9460-9444

\section{Marina de Lima Pinheiro}

Residente em Medicina de Família e Comunidade (MFC) pela Secretaria Municipal de Saúde de João Pessoa - SMS/JP em parceria com a Faculdade de Ciências Médicas da Paraíba (FCM-PB). Graduada em medicina pela Universidade Federal da Paraíba (UFPB).

ORCID: 0000-0001-9566-7366

\section{Melissa Maria Medeiros de Morais}

Residente em Medicina de Família e Comunidade (MFC) pela Secretaria Municipal de Saúde de João Pessoa - SMS/JP em parceria com a Faculdade de Ciências Médicas da Paraíba (FCM-PB). Graduada em Medicina pela Universidade Federal da Paraíba (UFPB).

ORCID: 0000-0003-2336-3907

\section{Maria Clara de Araujo Remigio Batista}

Graduanda do curso de Medicina da Unipê.

ORCID: 0000-0002-5345-890X

\section{INTRODUÇÃO}

A experiência do adoecimento tem impacto único na vida da pessoa. Pode ser forte e transformador, pode ser doloroso e incapacitante. Fato é que jamais iremos conhecer dois pacientes iguais, cada homem adoece a sua maneira. Nesse contexto o Diabetes mellitus (DM2), é entendido como sendo um problema de saúde pública, é visto como uma doença que afeta o sujeito, sua família e a comunidade. $\mathrm{O}$ diabetes tipo 2 é o mais comum, corresponde a $90 \%$ dos casos e é descrito como um "assassino silencioso". Em geral, é difícil determinar seu início exato, por mais que possam existir famílias com tendência a resistência à insulina. Até um terço a metade das pessoas podem não ser diagnosticadas ${ }^{1}$.

Associada a maiores taxas de hospitalizações e incapacitação física, devido às complicações macro e microvasculares, o DM2 é uma das doenças que faz maior utilização dos serviços de saúde, implicando em sobrecarga dos sistemas de saúde de todos os países, independentemente do seu desenvolvimento econômico. O pacientes por vezes estão desmotivados e albergam sentimentos conflitantes de desesperança com relação a saúde atual e futura $^{2}$.
Os objetivos mais importantes das ações de saúde em

DM2 são controlar

a glicemia, reduzir a morbi-mortalidade por complicaçôes cardiovasculares

e melhorar a

qualidade de vida

potencializando

e motivando os

pacientes a performar

o autocuidado.
Os objetivos mais importantes das ações de saúde em DM2 são controlar a glicemia, reduzir a morbi-mortalidade por complicações cardiovasculares e melhorar a qualidade de vida potencializando e motivando os pacientes a performar o autocuidado. A compensação da doença resulta da soma de diversos fatores e condições que propiciam o acompanhamento desses pacientes. Assim, uma intervenção educativa sistematizada, amorosa, empática e permanente é fundamental para mudar as práticas atuais ${ }^{3,4}$.

Identificar os fatores de risco, os sinais de lesão em órgão-alvo, sintomas de hiper ou hipoglicemia como os pacientes percebem sua doença e como aderem ao tratamento influencia diretamente no monitoramento glicêmico domiciliar ${ }^{5}$. Mesmo os pacientes diabéticos possuindo um conhecimento sobre esses temas, estudos apontam que itens relativos ao tratamento, como mudança do estilo de vida e alimentação, e o apoio familiar necessitam de incremento maior na educação ${ }^{6}$.

Embora sejam muitas as complicações sérias e dispendiosas que afetam os indivíduos com diabetes, tais como doenças do coração, problemas renais e cegueira, as complicações com os pé representam a maior parte: $40 \mathrm{a}$ $70 \%$ de todas as amputações das extremidades inferiores estão relacionadas ao diabetes 
mellitus, aproximadamente $85 \%$ de todas as amputações diabéticas eram precedidas por úlceras nos pés?.

O cuidado com o pé é essencial para reduzir o número de casos prejudicados por neuropatia e perda de sensibilidade periférica tátil, térmica e dolorosa que podem determinar lesões complexas que, quando não tratadas, podem evoluir com amputação do membro. Considera-se que o pé diabético seja consequência da infecção, ulceração e/ou destruição dos tecidos profundos, associados ao acometimento dos membros inferiores por diferentes graus de doença vascular periférica e por anormalidades neurológicas. Possui capacidade de reduzir a qualidade de vida do diabético, devido à possível amputação do membro afetado, sendo considerado uma causa comum de invalidez ${ }^{8}$.

No contexto de potencializar o cuidado uma ferramenta acessível à prática na Atenção Primária à Saúde (APS) é o Índice Tornozelo-Braquial (ITB), método importante na propedêutica da Doença Arterial Obstrutiva Periférica (DAOP), para o prognóstico de desfechos e mortalidade relacionados ao sistema cardiovascular?. Verifica-se inicialmente através da palpação dos pulsos de membros inferiores (artérias tibial posterior e pediosa) e de pulsos braquiais em membros superiores. Afere-se, então, a pressão arterial sistólica (PAS) destes locais com esfigmomanômetro e ecodoppler portátil. Escolhe-se o maior valor de PAS encontrado entre as artérias tibial posterior e pediosa e divide-se pelo da artéria braquial, bilateralmente ${ }^{10}$.

A APS inserida num contexto de descentralização e hierarquização das ações, ganha papel de destaque no cuidado do pé diabético. A Estratégia de Saúde da Família (ESF), a forma de incutir a APS no Sistema Único de Saúde (SUS) se configura como ferramenta na reorganização do sistema. Configura-se como um modelo de acompanhamento com atuação na integralidade e efetividade nos territórios e na oferta de serviços, visando ações de promoção, proteção e recuperação da saúde, devendo incorporar as mais diversas face- tas do complexo do quadro epidemiológico brasileiro ${ }^{11}$.

O aprimoramento contínuo da APS consegue obter resultados positivos em relação à adesão ao tratamento e à satisfação da população acerca dos tratamentos propostos. Estudos apresentaram evidências de que o cumprimento dos atributos dos serviços de APS está associado ao menor número de hospitalizações, ao menor número de consultas para um mesmo problema, à menor quantidade de exames complementares e à maior possibilidade de ações de prevenção ${ }^{12}$.

A qualidade na atenção prestada, medida pelos resultados na saúde da população e na redução das iniquidades em saúde, é mais uma meta a ser buscada. Lidamos ainda com lacunas, que vão desde a infraestrutura, passando pelos processos de trabalho, que viabilizam os princípios do acesso, da coordenação, da longitudinalidade e da integralidade, que comprometem a qualidade da atenção e a percepção do usuário. Tais entraves, além de sistemáticos e relevantes, são também evitáveis, injustos e desnecessários, devendo ser reduzidos ${ }^{12}$.

Diante dos inúmeros casos de diabéticos com complicações vasculares atendidos diariamente, nasceu a necessidade de abordar e reforçar a importância da avaliação dos pés diabéticos, para identificar de forma precoce as complicações microvasculares e macrovasculares. Além disto, trazer à tona a educação em saúde enquanto dispositivo para fortalecer a autoeficácia e motivação ao autocuidado, a partir do relato de experiência de residentes em Medicina de Família e Comunidade (MFC) na prática diária.

\section{MÉTOdOS}

Trata-se de um estudo observacional, descritivo e transversal, sob a forma de relato de experiência. Neste, três residentes de MFC, em conjunto com a preceptora responsável refletem acerca de suas experiências nos atendimentos diários a pacientes diabéticos, quanto ao papel da longitudinalidade como ferramenta potente no cuidado ao paciente portador de DM, sobretudo na avaliação do pé diabético.

O trabalho foi construído a partir do conjunto das vivências das residentes no suporte a essa população. Uma se encontra no primeiro ano de residência e duas no segundo, e estão vinculadas a duas equipes de ESF da Unidade de Saúde da Família (USF) Saúde e Vida Integrada, com aproximadamente 9000 usuários adscritos, localizada na cidade de João Pessoa (PB). A exploração dos conhecimentos e a troca das experiências, durante o seguimento dos casos, ocorreram em encontros mensais no período de Março a Setembro de 2020.

Durante as consultas de demanda espontânea, as residentes avaliavam feridas, glicemia e realizavam exame físico geral para identificar precocemente fatores que estivessem atrapalhando os resultados esperados, como falta de higiene, sapatos inadequados, condição dos pés, sensibilidade e a avaliação do Índice Tornozelo Braquial (ITB).

Por ser tratar de um relato de experiência das autoras deste trabalho, não houve necessidade de submissão ao Comitê de Ética em Pesquisa.

\section{RESULTADOS}

Um fator em comum no cotidiano das residentes foi a preponderância de pacientes portadores de DM2 acometidos por complicações micro ou macrovasculares identificados já na primeira consulta. Por vezes, deflagrou-se o sentimento de derrota, uma vez que os problemas em questão são evitáveis com táticas simples e factíveis.

O trabalho com uma equipe multiprofissional foi fundamental para acompanhar estes pacientes, somando forças para proporcionar um cuidado ampliado, com destaque para a enfermagem, as técnicas de enfermagem, a psicologia, a farmácia e a nutrição.

Ao decorrer dos meses, com a implementação de orientações, durante a consulta médica, quanto à importância do autocuidado e medidas básicas de avaliação de acometimento nos pés, notou-se 
significativa mudança no comportamento dos diabéticos avaliados. Onde antes existiam dificuldades na compreensão do processo de adoecimento, após algumas consultas observou-se maior discernimento quanto a este, com capacidade de prover o autocuidado.

Mesmo com uma realidade de recursos escassos na UBS, pode-se com o mínimo restaurar peles e até mesmo almas de pessoas que não possuíam autocuidado ou que não procuravam assistência à saúde. A longitudinalidade como uma das características da APS permitiu uma melhora do acesso aos usuários e, consequentemente, da qualidade de vida destes.

A partir dessas vivências, tornou-se como rotina no cuidado aos diabéticos tanto o exame físico do pé, como também a avaliação do ITB. Além disso, foram discutidas atualizações sobre cuidados de feridas e equipamentos com a preceptoria para capacitar melhor as residentes de MFC. Ao fim, percebeu-se uma maior independência dos diabéticos após início do cuidado intensificado.

\section{DISCUSSÃO}

O Brasil tem passado por grandes mudanças, nos últimos anos, na estrutura do SUS. O Programa Saúde da Família (PSF), por exemplo, posteriormente ampliado para a ESF, foi desenvolvido com a finalidade de melhorar o acesso à APS e a qualidade desta em todo o país. Seu caráter estruturante dos sistemas municipais de saúde tem provocado um importante movimento com o intuito de reordenar o modelo de atenção no $S U S^{13}$.

Entretanto, mesmo diante de reformas implantadas, a incidência de DM2 encontra-se em ascensão, associada a um alto custo econômico e social. A alteração desse quadro passa obrigatoriamente pela organização e incentivo da $A B$, que se têm mostrado menos dispendiosa, garantindo o diagnóstico e o acesso aos diferentes tratamentos, e possibilita a implantação de medidas que retardam o aparecimento da doença ou evitam suas complicações ${ }^{14}$. Deste modo, é imprescindível a vincula- ção do paciente diabético às unidades de saúde da família ${ }^{15}$.

\section{O conhecimento}

da patologia possui

ação preponderante

na potencialização

do autocuidado,

uma vez que

cada paciente é

um universo e

experiência de

maneiras diferentes

o processo de

adoecimento.

O tratamento deve avaliar os fatores e a individualidade de cada indivíduo, dependendo do caso e dos alvos glicêmicos ${ }^{16}$. A euglicemia se apresenta como um entrave para o cuidado eficaz dos diabéticos, tendo o empoderamento destes uma função primordial para alcançar um melhor controle de sua doença ${ }^{17}$. Uma estratégia que inclui prevenção, educação de profissionais e do paciente, tratamento multifatorial da ulceração e rígida monitorização podem reduzir as taxas de amputação de 49 a $85 \%$.
A amputação de um membro, decorrente de uma doença de início insidioso com potencial avassalador, em uma pessoa ativa e sem deformidades gera impactos psicológicos importantes - como depressão, isolamento social e redução da autoestima - tendo de se adaptar a uma condição que lhe foi imposta e lidar com os julgamentos próprios e alheios ${ }^{18}$.

Quanto à equipe de $\mathrm{AB}$, cabe identificar e tratar as lesões pré-ulcerativas e deformidades de baixo risco, além de buscar fatores do cotidiano que possam influenciar em complicações futuras ${ }^{19}$. Em relação ao tratamento, busca-se deixar a ferida limpa, úmida e coberta para facilitar o processo de cicatrização, para isso, é crucial observar e diferenciar a presença de tecidos viáveis e inviáveis (para possíveis desbridamentos) e realizar a troca de curativos diariamente, orientando o paciente $\mathrm{e}$ o cuidador sobre a técnica ${ }^{20}$.

Os procedimentos realizados na APS incluem remoção de calos, fissuras, cortes de unhas (no caso de alterações ungueais, deve-se avaliar a necessidade de cantoplastia), tratamento de onicomicoses e lesões neuro-isquêmicas, avaliação periódica do pé de maior risco e da diminuição da sensibilidade plantar, manejo de úlceras não complicadas $^{20}$.

A educação em saúde no autocuidado dos portadores de pé diabético contribui significativamente para diminuição de agravos, incluindo a inspeção diária dos pés, a higiene, a hidratação, a secagem dos espaços interdigitais e o estímulo ao uso de calçados adequados com exame minucioso ao colocá-los ${ }^{21}$.

\section{CONCLUSÃO}

As expectativas oriundas dos profissionais ante ao controle de danos em pacientes com doenças crônicas muitas vezes tornam o cuidado desafiador, principalmente quando acrescidas das idealizações dos pacientes.

O conhecimento da patologia possui ação preponderante na potencialização do autocuidado, uma vez que cada paciente é um universo e experiência de maneiras diferentes o processo de adoecimento. 


\section{artigo}

Fernandes, D.M.A.P.; Melo, V.F.C.; Assis, L.M.B.; Pinheiro, M.L.; Morais, M.M.M.; Batista, M.C.A.R.

A insustentável leveza do toque: reconhecimento do cuidado ao pé diabético

Por ser complicação frequente e potencialmente grave, porém evitável, o pé dia- bético deve ser parte da rotina de acompanhamento dos pacientes com DM2, visto que a APS consegue agir com eficácia no tratamento e prevenção deste.

\section{REFERÊNCIAS}

1.International Diabetes Federation. IDF DIABETES ATLAS, 9th edn. Brussels, Belgium: 2019. [cited 2020 Nov 26]. Available from: https:/www.diabetesatlas.org/en/

2.Sociedade Brasileira de Diabetes. Diretrizes Sociedade Brasileira de Diabetes 2017-2018. São Paulo: 2017. [cited 2020 Nov 26]. Available from: https:/www.diabetes.org.br/profissionais/images/2017/diretrizes/diretrizes-sbd-2017-2018.pdf

3.Brasil. Ministério da Saúde. Secretaria de Atenção à Saúde. Departamento de Atenção Básica. Estratégias para o cuidado da pessoa com doença crônica: diabetes mellitus / Ministério da Saúde, Secretaria de Atenção à Saúde, Departamento de Atenção Básica. - Brasilia: Ministério da Saúde, 2013.

4.Araujo LM, Britto MM, Cruz TR. Tratamento do diabetes mellitus do tipo 2: novas opções. Arq Bras Endocrinol Metab, São Paulo, v. 44, n. 6, p. 509-518, Dec. 2000. Available from: <http:/www.scielo.br/scielo.php?script=sci_arttext\&pid=S0004-27302000000600011\&Ing=en\&nrm=iso>. access on 27 Jan. 2020 . http://dx.doi. org/10.1590/S0004-27302000000600011.

5.Oliveira LL, Lima GF, Nunes TS, Moura TA, Tanajura DM. Atitudes e comportamentos dos diabéticos acerca das estratégias de prevenção e controle clínico do diabetes/Attitudes and behaviors of diabetics about the diabetes clinical prevention and control strategies. Rev Med (São Paulo). 2019 jan.-fev.;98(1):16-22. Available from: https://www.revistas.usp.br/revistadc/article/view/148752

6. Chagas IA, Camilo J, Santos MA, Rodrigues FF, Arrelias CC, Teixeira $\mathrm{CR}$ et al. Conhecimento de pacientes com diabetes sobre tratamento apos cinco anos do término de um programa educativo. Rev. esc. enferm. USP [Internet]. 2013 [cited 2020 Nov 26]; 47(5): 1137-42. Available from http:/www.scielo.br/scielo.php?script=sci_arttext\&pid=S0080-62342013000501137

7. Secretaria de Estado de Saúde do Distrito Federal. Consenso Internacional sobre pé diabético. Brasília: Imprensa Oficial; 1999. Available from: http://189.28.128.100/dab/docs/publicacoes/geral/conce_inter_pediabetico.pdf

8. Sampaio LF, Mendonça CS, Turci MA. Atenção primária à saúde no Brasil. In: Gusso G, Lopes JM, Dias LC. Tratado de Medicina da Familia e Comunidade. 2. ed. Porto Alegre: Editora Artmed; 2019. v.1, p 273.

9. Kawamura T. Índice Tornozelo-Braquial (ITB) determinado por esfigmomanômetros oscilatórios automáticos. Arq Bras Cardiol. 2008 [11 jun 2020]; Available from: https://www.scielo.br/scielo.php?pid $=$ S0066-782X2008000500003\&script=sci_abstract\&tlng=pt

10. Dimarzio G, Yoshida RA. Doença Arterial Periférica. In: Gusso G, Lopes JMC, Dias LC. Tratado de Medicina de Familia e Comunidade. 2. ed. Porto Alegre: Editora Artmed; 2019. v.1, p 4194-4219.

11. Ministério da Saúde (BR), Secretaria de Assistência à Saúde. Saúde da Familia: uma estratégia para reorganização do modelo assistencial. Brasilia (DF), 1997. Available from: http://bvsms.saude. gov.br/bvs/publicacoes/cd09_16.pdf
12. Cubas MR, Santos OM, Retzlaff EM, Telma HL, Andrade IP, Moser AD, et al. Pé diabético: orientações e conhecimento sobre cuidados preventivos. FisioterMov. [Internet]. 2013 [cited 2020 Nov 26];26(3):647-655. Available from: https://www.scielo.br/pdf/fm/ v26n3/a19v26n3.pdf

13. Alfradique ME, Bonolo PF, Dourado I, Lima-Costa MF, Macinko J, Mendonça, CS, et al. Internaç̧ões por condições sensiveis à $\quad$ atenção primária: a construção da lista brasileira como ferramenta para medir o desempenho do sistema de saúde (Projeto ICSAP - Brasil). Cad Saúde Pública. [Internet] 2009 [cited 2020 Nov 26];25(6):1337-49. Available from: http://dx.doi. org/10.1590/S0102-311X2009000600016

14. Guidoni CM, Oliveira CM, Freitas O, Pereira LR. Assistência ao diabetes no Sistema Único de Saúde: análise do modelo atual. BrazJPharmSci. [Internet] 2009 [cited 2020 Nov 26];45(1): 37-48. Available from: http://www.scielo.br/pdf/bjps/v45n1/05.pdf

15. MINISTÉRIO DA SAÚDE (BR), Secretaria de Vigilância em Saúde. A vigilância, o controle e a prevenção das doenças crônicas não-transmissíveis: DCNT no contexto do Sistema Único de Saúde brasileiro. Brasîlia (DF); 2005. Available from: https://bvsms.saude. gov.br/bvs/publicacoes/DCNT.pdf

16. Pharmacologic Approaches to Glycemic Treatment: Standards of Medical Care in Diabetes-2019. Diabetes Care [Internet] 2019[cited 2020 Nov 26];42(Suppl.1): S90-S102. Available from: https:/doi. org/10.2337/dc19-S009

17. Santos MM, Peters AA, Figueiredo MA, Carreiro MA, Magalhães MC, Araújo EP. Controle glicêmico, suporte social percebido e o autocuidado de indivíduos com diabetes tipo 2. SaudColetiv. [Internet]. 2020 [cited 2020 Nov 26];10(55):2815-2822. Available from: https://doi.org/10.36489/saudecoletiva.2020v10i55p2815-2830

18. Silva FM, Viana MC, Barreto JO, Sousa NM, Penha AA. Síntese de evidências para políticas de saúde: prevenção e controle do pé diabético na atenção primária à saúde. Bis, São Paulo, v. 20, n.2, p.7788, dez. 2019. Available from: https://pesquisa.bvsalud.org/portal/ resource/pt/biblio-1022213

19. Nascimento MT, Silva NI, Brito FC, Fontes FL, Oliveira AF, OIiveira JV, et al. Fatores de risco associados ao desenvolvimento do pé diabético e ações executadas na Atenção Primária à Saúde para prevenção do agravo. REAS [Internet]. 2019 [cited 2020 Nov 26];(33):e1371. Available from: https:/acervomais.com.br/index. php/saude/article/view/1371

20. Ministério da Saúde (BR), Secretaria de Atenção à Saúde. Manual do Pé Diabético: estratégias para o cuidado da pessoa com doença crônica. Brasilia, 2016. Available from: http://www.as.saude.ms.gov. br/wp-content/uploads/2016/06/manual_do_pe_diabetico.pdf

21. Mendes RN, Lisboa MS, Lima TP. Atuação do Enfermeiro no Autocuidado com o Paciente com Diabetes Mellitus Tipo II e Pé Diabético. Revista Multidisciplinar e de psicologia [Internet]. 2020 [cited 2020 Nov 26]. Available from: 10.14295/idonline.v14i51.2565. 